Blühdorn, H. - Syntaktische Nebensatzklassen im Deutschen

\title{
Syntaktische Nebensatzklassen im Deutschen
}

\author{
[Syntactic classes of subordinate clauses in German]
}

Hardarik Blühdorn ${ }^{1}$

\begin{abstract}
This article gives an overview of the syntactic classes of subordinate clauses in German. Their functions and structures are described with the help of the model of linear positions and fields. Three questions are treated in more detail: the functions of (i) pronouns and (ii) comparative particles in relation to subordinate clauses and (iii) the role of subordinate clauses in cleft constructions. The article can be used in academic teaching and as a starting point for studies in comparative syntax.
\end{abstract}

Keywords: Syntax of German; subordinate clauses; pronouns; comparative particles; cleft constructions.

Resumo: O artigo dá uma visão panorâmica das classes de orações subordinadas da língua alemã. Suas funções e estruturas sintáticas são descritas mediante o esquema das posições e dos campos. Três aspectos específicos são tratados mais a fundo: as funções (i) de pronomes e (ii) de partículas de comparação em relação a orações subordinadas e (iii) o papel de orações subordinadas em construções clivadas. $\mathrm{O}$ artigo pode ser usado para fins didáticos no meio acadêmico e como ponto de partida para estudos em sintaxe comparativa.

Palavras-chave: Sintaxe da língua alemã; orações subordinadas; pronomes; partículas de comparação; construções clivadas.

Zusammenfassung: Der Aufsatz gibt einen Überblick über die Arten von Nebensätzen im Deutschen. Er beschreibt ihre syntaktischen Funktionen und Strukturen anhand des Felderschemas. Auf drei Gesichtspunkte wird detaillierter eingegangen: nebensatzspezifische Funktionen von (i) Pronomina und (ii) Vergleichspartikeln sowie (iii) die Rolle von Nebensätzen bei der Satzspaltung. Der Aufsatz kann im Universitätsunterricht und als Grundlage für Untersuchungen in vergleichender Syntax genutzt werden.

Stichwörter: Syntax des Deutschen; Nebensätze; Pronomina; Vergleichspartikeln; Satzspaltung.

\footnotetext{
${ }^{1}$ Wissenschaftler am Institut für Deutsche Sprache Mannheim; Prof. Livre-Docente (Universität São Paulo), Email: hardarik@ids-mannheim.de.
} 
Blühdorn, H. - Syntaktische Nebensatzklassen im Deutschen

\section{Einleitung}

Der vorliegende Aufsatz ${ }^{2}$ entwickelt eine syntaktische Klassifikation der deutschen Nebensätze aufgrund von zwei Kriterien:

(i) ihrer syntaktischen Funktion innerhalb des Satzes, dessen Konstituente sie sind, und

(ii) ihrer internen syntaktischen Struktur.

Ziel ist es, einen Überblick über die Nebensatzarten des Deutschen zu geben, der im Universitätsunterricht genutzt und sprachvergleichenden grammatischen Untersuchungen zugrundegelegt werden kann.

Unter einem Nebensatz verstehe ich einen satzförmigen Ausdruck, der als Konstituente eines komplexeren Satzes (eines Matrixsatzes) auftritt. Satzförmig heißen Ausdrücke, die ein finites Verb als syntaktischen Kern enthalten. Im Deutschen enthalten solche Ausdrücke in aller Regel zugleich ein explizites Subjekt. Nicht-satzförmige verbhaltige Ausdrücke wie Infinitiv- oder Partizipialgruppen (sogenannte satzwertige Ausdrücke; vgl. DUDEN 2009: 852ff.; BLÜHDORN 2012c) klammere ich aus. Meine Darstellung stützt sich auf die IDS-Grammatik (ZIFONUN et al. 1997), die aktuelle Auflage der Duden-Grammatik (DUDEN 2009) sowie auf BLÜHDORN / LOHNSTEIN (2012) und die dort verarbeitete weitere Literatur.

Typische Nebensätze des Deutschen sind durch die Endstellung des finiten Verbs (VE-Stellung) gekennzeichnet. In Hauptsätzen ist das Finitum demgegenüber meist vorangestellt, d.h. es nimmt entweder die erste (V1-Stellung) oder die zweite Linearposition (V2-Stellung) ein. Dieses Unterscheidungskriterium gilt aber nicht ausschließlich. Es gibt durchaus Sätze mit VE-Stellung, die man aufgrund ihrer syntaktisch und pragmatisch selbständigen Verwendung als Hauptsätze einstufen möchte, etwa Wunschsätze vom Typ (1), Ausrufesätze vom Typ (2) oder nachdenkliche Fragen vom Typ (3):

\footnotetext{
${ }^{2}$ Für wertvolle Hinweise danke ich Reinhard Fiehler, Marina Foschi Albert und Bernd Wiese.
} 
Blühdorn, H. - Syntaktische Nebensatzklassen im Deutschen

(1) Wenn ich nur einen Euro hätte!

(2) Dass ich das noch erleben darf!

(3) Ob das wohl schon alles war?

Pragmatisch selbständig ist ein Satz, wenn mit ihm ein vollständiger und abgeschlossener Sprechakt ausgeführt wird. Dafür sind Sätze wie (1) bis (3) zweifellos geeignet. Syntaktisch selbständig ist ein Satz, der nicht als Konstituente eines komplexeren Satzes auftritt. Sätze wie (1) bis (3) können syntaktisch selbständig, daneben aber auch syntaktisch unselbständig, also als Konstituenten komplexerer Sätze (und damit als Nebensätze) gebraucht werden:

(1a) Wenn ich nur einen Euro hätte, würde ich mir das Leben nehmen.

(2a) Dass ich das noch erleben darf, hätte ich nicht gedacht.

(3a) Er fragte sich, ob das wohl schon alles war.

Allgemein gilt: Jeder VE-Satz des Deutschen kann als Nebensatz verwendet werden; manche VE-Sätze können auch als Hauptsätze verwendet werden (insbesondere Komplementierersätze und indirekte w-Fragesätze; s.u. Abschnitte 2.2 und 2.3). VE-Sätze, die nur als Hauptsätze verwendbar sind, gibt es nicht.

Umgekehrt verhält es sich mit V1- und V2-Sätzen. Sie werden typischerweise syntaktisch und pragmatisch selbständig verwendet, lassen daneben aber auch syntaktisch unselbständige Verwendungsweisen zu, wie wir im weiteren Verlauf sehen werden (s.u. Abschnitte 2.4, 2.6, 3.3, 4.3, 5.2.2 und 5.6). Hauptsätze und Nebensätze sind im Deutschen also letztlich nicht aufgrund ihrer internen syntaktischen Struktur zu unterscheiden, sondern aufgrund ihrer externen syntaktischen Funktion: Syntaktisch (und pragmatisch) selbständige Sätze sind Hauptsätze, syntaktisch unselbständige Sätze sind Nebensätze. Pragmatische Gesichtspunkte werde ich im Folgenden nur noch am Rande in Betracht ziehen und mich weitgehend auf die Syntax konzentrieren.

Unter den Konstituenten von Sätzen wird üblicherweise (vgl. z.B. DUDEN 2009: 795f.) zwischen Argumenten/Aktanten (nach Art und Anzahl durch das Verb oder einen anderen Prädikatsausdruck selegierten Satzgliedern; prototypisch: Subjekten und Objekten), Prädikativen (Satzgliedern, die durch eine Kopularelation mit einem Argument verbunden sind) und Adverbialia (frei hinzufügbaren Satzerweiterungen) unterschieden. Diese drei 
Blühdorn, H. - Syntaktische Nebensatzklassen im Deutschen

Gruppen werden als Satzglieder zusammengefasst. Für die Beschreibung der internen Struktur von (vor allem nominalen) Satzgliedern spielt außerdem der Sammelbegriff des Attributs eine wichtige Rolle, mit dem Erweiterungen zu Satzgliedern und Satzgliedkernen erfasst werden.

In den folgenden Kapiteln betrachte ich satzförmige Konstituenten aller dieser Klassen und stelle die Möglichkeiten dar, die jeweiligen syntaktischen Funktionen durch Sätze unterschiedlicher Strukturtypen zu realisieren. Als Vertiefungsaspekte, die an mehreren Stellen wichtig werden, behandle ich nebensatzspezifische Funktionen von Pronomina und Vergleichspartikeln sowie die Rolle von Nebensätzen bei der Satzspaltung.

\section{Argumentsätze}

Als Argumentsätze fasse ich satzförmig realisierte Subjekte und Objekte sowie Ergänzungen zu adjektivischen Prädikaten, zu Gradprädikaten und zu Vergleichsprädikaten zusammen. Sie stehen im Allgemeinen im Vor- oder Nachfeld des Matrixsatzes, nur ausnahmsweise in dessen Mittelfeld (vgl. ZIFONUN et al. 1997: 1651). Als Argumente kommen Sätze aller Strukturtypen in Frage: freie Relativsätze, indirekte w-Fragesätze, Komplementierersätze, V2Sätze, Vergleichspartikelsätze und V1-Sätze (vgl. ZIFONUN et al. 1997: 1448f.).

\subsection{Freie Relativsätze}

Eine satzförmige Realisierungsmöglichkeit, die für praktisch jede Subjekt- und Objektstelle (und überhaupt für fast jede Satzgliedklasse) gegeben ist, ist der freie Relativsatz. Es handelt sich um einen VE-Satz, der durch eine Relativphrase, meist einen relativen w-Ausdruck, eingeleitet wird und der nicht als Erweiterung zu einem Bezugsausdruck fungiert:

\section{Wer kein Geld hat, muss essen, was auf den Tisch kommt.}

In Beispiel (4) sind Subjekt und Akkusativobjekt zu muss essen als freie Relativsätze realisiert. Solche Sätze werden häufig generalisierend im Sinne von 'jeder, der p' oder 'alles, was p' verwendet (vgl. ENGEL 1996: 248ff.; ZIFONUN et al. 1997: 2264ff.). Der einleitende 
Blühdorn, H. - Syntaktische Nebensatzklassen im Deutschen

w-Ausdruck steht für eine Menge von Alternativen, für die der Satz gleichermaßen gültig sein soll (vgl. BLÜHDORN/LOHNSTEIN 2012: 194f.). Freie Relativsätze können aber auch in spezifischer Lesart verwendet werden, und zwar dann, wenn der Sprecher das Gemeinte nicht kategorisieren, sondern nur über die Teilnahme an einem Sachverhalt beschreiben will:

$$
\text { Otto aß gerade, was auf seinem Teller lag. }
$$

In (5) ist der freie Relativsatz bevorzugt als Ausdruck für einen spezifischen Referenten zu lesen, der in einem bestimmten Moment von Otto verzehrt wird. Der Sprecher sagt über diesen Referenten - salopp gesprochen -, was er tut (auf dem Teller liegen), nicht dagegen, was er ist (z.B. ein Fisch).

Die interne syntaktische Struktur von Relativsätzen wird heute allgemein so gesehen, dass der einleitende Relativausdruck im Vorfeld des Relativsatzes steht:

\begin{tabular}{l|l|l|l|l} 
Vorfeld & $\begin{array}{l}\text { linke Klammer- } \\
\text { position }\end{array}$ & Mittelfeld & $\begin{array}{l}\text { rechte Klammer- } \\
\text { position }\end{array}$ & Nachfeld \\
\hline wer & & kein Geld & hat \\
was & & auf den Tisch & kommt &
\end{tabular}

Abb. 1: Felderstruktur freier Relativsätze

Diese Sicht unterscheidet sich von der älteren Annahme, derzufolge der Relativausdruck die linke Satzklammerposition einnimmt (vgl. z. B. ENGEL 1996: 305, 708). Das entscheidende Argument für die Analyse in Abb. 1 liegt darin, dass Relativausdrücke Phrasen sind, die syntaktisch erweitert werden können (vgl. etwa STERNEFELD 2008/09: 362ff.):

Wer von den Gästen zu spät kommt, muss stehen.

\begin{tabular}{l|l|l|l|l} 
Vorfeld & linke Klammer & Mittelfeld & rechte Klammer & Nachfeld \\
\hline wer von den Gästen & & zu spät & kommt &
\end{tabular}

Abb. 2: Freier Relativsatz mit Mehrwort-Relativum 
Blühdorn, H. - Syntaktische Nebensatzklassen im Deutschen

Der Relativausdruck in (6) ist die Nominalgruppe wer von den Gästen. Phrasen sind in der linken Satzklammerposition nicht zugelassen. Sie ist eine Position, in der nur syntaktische Köpfe stehen dürfen, die durch den Rest des Satzes ergänzt werden, nämlich unterordnende Konjunktionen oder finite Verbformen. Das Vorfeld hingegen ist eine Phrasenposition. Relativsätze haben demnach ein besetztes Vorfeld und eine unbesetzte linke Klammerposition. Bei konjunktional eingeleiteten Nebensätzen ist es umgekehrt (s.u. Abschnitte 2.3, 2.5 und 4.2).

Freien Relativsätzen kann ein d-Pronomen als Bezugsausdruck vorangestellt werden. Dann sind sie nicht mehr frei, sondern fungieren als Attribut zu diesem Pronomen (s.u. Abschnitt 5.2.1). In (4a) steht der Subjektsrelativsatz zusammen mit dem d-Pronomen im Vorfeld. Das Bezugspronomen des Objektsrelativsatzes steht im Mittelfeld; der Objektsrelativsatz selbst ist ausgeklammert (steht im Nachfeld):

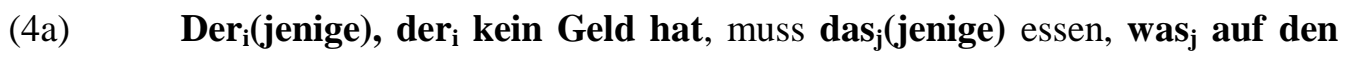
Tisch kommt.

Die Satzbedeutung wird durch die Umwandlung freier in gebundene Relativsätze nicht verändert (vgl. LEHMANN 1984: 299). Sie wird aber expliziter ausgedrückt. Nach Bezugspronomina im Maskulinum, Femininum oder Plural - der(jenige), die(jenige), die(jenigen) - steht in gebundenen Relativsätzen anstelle des w-Relativums wer das dRelativum der bzw. die.

Freie Relativsätze können auch (ebenso wie Ausdrücke anderer Klassen) durch ein nachfolgendes koindiziertes ${ }^{3}$ Pronomen wiederaufgenommen werden:

(4b) [Wer kein Geld hat $]_{\mathrm{i}}$, der $_{\mathrm{i}}$ muss essen, was auf den Tisch kommt.

Solche Pronomina werden als Resumptiva bezeichnet (vgl. EISENBERG 2004: 329). Die wiederaufgenommene Konstituente ist ihr Antezedens. Antezedens und Resumptivum haben die gleiche syntaktische Funktion. Es gibt unterschiedliche Meinungen darüber, wie sie sich

\footnotetext{
${ }^{3}$ Ich verwende Indizes, um Ausdrücke gleicher syntaktischer und/oder gleicher referentieller Funktion sowie syntaktische Variable und ihre Bezugsausdrücke zu kennzeichnen.
}

Pandaemonium, São Paulo, v. 16, n. 21, Jun/2013, p.149-189 www.fflch.usp.br/dllm/alemao/pandaemoniumgermanicum 
Blühdorn, H. - Syntaktische Nebensatzklassen im Deutschen

in der Satzstruktur zueinander verhalten. Eine verbreitete Analyse nimmt für Sätze wie (4b) an, dass das Antezedens nach links in eine Position vor dem Vorfeld des Matrixsatzes versetzt wurde und das Resumptivum das Vorfeld einnimmt (vgl. ALTMANN 1981: 47f.; ZIFONUN et al. 1997: 518f.):

\begin{tabular}{l|l|l|l|l|l} 
Vorvorfeld & Vorfeld & $\begin{array}{l}\text { linke } \\
\text { Klammer }\end{array}$ & Mittelfeld & $\begin{array}{l}\text { rechte } \\
\text { Klammer }\end{array}$ & Nachfeld \\
\hline wer kein Geld hat, & der & muss & & essen, & $\begin{array}{l}\text { was auf den Tisch } \\
\text { kommt }\end{array}$
\end{tabular}

Abb. 3: Felderanalyse für (4b) mit „Linksversetzung“

Meines Erachtens gibt es gute Argumente für die alternative Annahme, dass Antezedens und Resumptivum in Sätzen wie (4b) gemeinsam im Vorfeld stehen:

\begin{tabular}{l|l|l|l|l} 
Vorfeld & LK & Mittelfeld & RK & Nachfeld \\
\hline wer kein Geld hat, der & muss & & essen, & was auf den Tisch kommt
\end{tabular}

Abb. 4: Alternative Felderanalyse für (4b)

Die Frage ist komplex. Die Einzelheiten können hier nicht weiter diskutiert werden.

Freie Relativsätze treten in bestimmten Arten von Spaltsätzen (vgl. REICHMANN 2005; BLÜHDORN 2012a: 194ff.) auf, und zwar bei der Abspaltung von Verbalphrasen. Die abgespaltene Verbalphrase ist dann mit dem Relativum koindiziert. Ich setze den freien Relativsatz fett und die abgespaltene Verbalphrase kursiv:

Was $_{\mathrm{i}}$ Otto wollte, war [sein Auto waschen $]_{\mathrm{i}}$.

Ob der Relativsatz in solchen Konstruktionen als Subjekt oder als Prädikativ zum Verb sein fungiert, ist nicht sicher entscheidbar. Für die Erkennung des Subjekts sind mehrere Kriterien relevant. Im Deutschen steht die Kongruenz mit dem finiten Verb im Vordergrund. In Sätzen mit Verbalphrasen-Abspaltung zeigt aber keines der beiden Satzglieder Kongruenz. In (7a) ist der freie Relativsatz Singular; die abgespaltene Verbalphrase kann aufgrund der Koordination 
Blühdorn, H. - Syntaktische Nebensatzklassen im Deutschen

als Plural gedeutet werden (vgl. EISENBERG 2004: 289); das Finitum steht im Singular. In (7b) kann der freie Relativsatz als Plural gedeutet werden; die abgespaltene Verbalphrase ist Singular; das Finitum steht wiederum im Singular:

(7a) Was S $_{\mathrm{i}}$ Otto wollte, war (*waren) [sein Auto waschen und seinen Hund füttern $]_{\mathrm{i}}$.

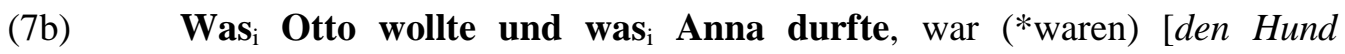
füttern $]_{\mathrm{i}}$.

Ein zweites Kriterium für die Subjekterkennung ist die lineare Abfolge der Satzkonstituenten. Auch hinsichtlich dieses Kriteriums sind Sätze mit Verbalphrasen-Abspaltung nicht festgelegt. Der freie Relativsatz kann im Vorfeld stehen wie in (7a) und (7b) oder im Nachfeld wie in (7c). Bei Nachstellung geht dem Relativsatz jedoch typischerweise ein koindiziertes Korrelatpronomen es voraus:

(7c) $\quad[\text { Sein Auto waschen }]_{\mathrm{i}}$ ist es $\mathrm{e}_{\mathrm{j}}$ gewesen, $\left[\text { was }_{\mathrm{i}} \text { Otto wollte }\right]_{\mathrm{j}}$.

Korrelatpronomina (vgl. EISENBERG 2004: 328ff.) fungieren, anders als vorausgehende koindizierte d-Pronomina, nicht als Bezugsausdrücke für nachfolgende Relativsätze. Sie sind syntaktische Variable und zeigen eine grammatische Position an, die für die Interpretation eines ausgeklammerten Nebensatzes relevant ist. Das Auftreten von Korrelatpronomina kann als Hinweis darauf gedeutet werden, dass freie Relativsätze bei Verbalphrasen-Abspaltung als Subjekte fungieren (vgl. ZIFONUN et al. 1997: 1082, 1475ff.). Die abgespaltene Verbalphrase behält unter dieser Analyse den ihr intuitiv zukommenden Prädikatsstatus.

\subsection{Indirekte w-Fragesätze}

Indirekte w-Fragesätze kommen hauptsächlich als Objektsätze in Betracht wie in (8). Sie können aber auch als Subjektsätze fungieren wie in (9):

(8) Otto soll feststellen, was vom Bankett übriggeblieben ist.

(9) Was vom Bankett übriggeblieben ist, ist uns noch nicht bekannt. 
Blühdorn, H. - Syntaktische Nebensatzklassen im Deutschen

Der Interrogativausdruck, der einen indirekten w-Fragesatz einleitet, ist eine Phrase, kann erweitert werden und muss deshalb, ebenso wie ein Relativausdruck, im Vorfeld stehen. Die linke Klammerposition indirekter w-Fragesätze ist leer:

\begin{tabular}{l|l|l|l|l} 
VF & LK & MF & RK & NF \\
\hline was & & vom Bankett & übriggeblieben ist &
\end{tabular}

Abb. 5: Felderstruktur indirekter w-Fragesätze

Indirekte w-Fragesätze sind strukturell von freien Relativsätzen nicht unterscheidbar. Auch funktional ist die Unterscheidung schwierig. Trotzdem wird allgemein an ihr festgehalten (z. B. DUDEN 2009: 1037ff., 1042ff.). Während freie Relativsätze unabhängig vom Verb in praktisch jeder Subjekt- und Objektstelle zugelassen und überdies auch in anderen Satzgliedfunktionen verwendbar sind, werden als indirekte w-Fragesätze nur Argumentsätze zu Prädikatsausdrücken bezeichnet, deren Bedeutung auf eine Wissenslücke hinweist, die durch eine Mitteilung geschlossen werden kann (vgl. EnGEL 1996: 248). Demnach ist der Nebensatz in (8) als indirekter w-Fragesatz, der in (10) als freier Relativsatz zu deuten:

(8) Otto soll feststellen, was vom Bankett übriggeblieben ist.

(10) Otto soll essen, was vom Bankett übriggeblieben ist.

ZIFONUN et al. (1997: 2264ff.) haben darauf hingewiesen, dass freie Relativsätze in spezifischer Lesart für Gegenstände bzw. in generalisierender Lesart für Mengen von Gegenständen stehen, während indirekte w-Fragesätze für Aussagen stehen. So steht der Nebensatz in (10) für die Menge der Dinge, die vom Bankett übriggeblieben sind, der in (8) dagegen für die Aussage 'vom Bankett ist x übriggeblieben'.

Ein recht deutlicher Unterschied zwischen indirekten Fragesätzen und freien Relativsätzen, die nicht durch Verbalphrasen-Abspaltung entstanden sind (s.o. Abschnitt 2.1), zeigt sich bei der Auswahl vorausgehender Proformen zu Subjektsätzen. Indirekten Fragesätzen in Subjektfunktion muss, wenn sie nachgestellt werden, ein Korrelatpronomen es vorausgehen, das mit dem Fragesatz als ganzem koindiziert ist. Relativsätzen kann nur ein dPronomen vorangestellt werden, das als Bezugsausdruck für das Relativum gedeutet werden 
Blühdorn, H. - Syntaktische Nebensatzklassen im Deutschen

muss. Betrachten wir dazu ein Beispiel. Der Subjektsatz in (11), dem kein Pronomen vorausgeht, kann entweder als indirekter Fragesatz oder als freier Relativsatz gelesen werden:

(11) Was du gesagt hast, ist mir egal.

Liest man ihn als indirekten Fragesatz, so bedeutet der Gesamtsatz: 'Es ist mir nicht wichtig, zu wissen, welche Äußerung du gemacht hast'. Diese Lesart kann auch durch (11a), nicht aber durch (11b) oder (11c) ausgedrückt werden. Liest man den Subjektsatz als freien Relativsatz, so bedeutet der Gesamtsatz: 'Die Äußerung, die du gemacht hast, bzw. ihr Inhalt ist mir nicht wichtig'. Diese Lesart kann nicht durch (11a), wohl aber durch (11b) und (11c) paraphrasiert werden. In $(11 \mathrm{~b} / \mathrm{c})$ ist der Relativsatz Attribut zum vorangestellten d-Pronomen (s. auch u. Abschnitt 5.2.1):

(11a) Es $s_{\mathrm{i}}$ ist mir egal, [was du gesagt hast $]_{\mathrm{i}}$.

(11b) Das (jenige), was $_{\mathrm{i}}$ du gesagt hast, ist mir egal.

(11c) Mir ist das $_{\mathrm{i}}$ egal, was $\mathrm{s}_{\mathrm{i}}$ du gesagt hast.

\subsection{Komplementierersätze}

Die häufigsten Beispiele für Subjekt- und Objektsätze in Grammatiken und Lehrbüchern sind dass-Sätze. Ebenso wie indirekte w-Fragesätze sind sie nur für bestimmte Argumentstellen bestimmter Verben zugelassen (vgl. ZIFONUN et al. 1997: 1448ff.):

*Dass Otto immer noch über der Pizzeria wohnt, muss essen, dass sie dort gute Pizza backen.

Dass Otto immer noch über der Pizzeria wohnt, muss bedeuten, dass sie dort gute Pizza backen.

Beispiel (12) zeigt, dass das Verb essen keine dass-Sätze als Subjekt oder Objekt zulässt. (13) zeigt, dass das Verb bedeuten in beiden Funktionen solche Sätze zulässt.

Wörter wie dass stehen im Unterschied zu Relativ- und Interrogativausdrücken nicht im Vorfeld, sondern in der linken Klammerposition des von ihnen eingeleiteten Nebensatzes: 
Blühdorn, H. - Syntaktische Nebensatzklassen im Deutschen

\begin{tabular}{l|l|l|l|l}
$\mathbf{V F}$ & $\mathbf{L K}$ & $\mathbf{M F}$ & $\mathbf{R K}$ & $\mathbf{N F}$ \\
\hline & dass & Otto kein Geld & hat & \\
& ob & Anna verheiratet & ist &
\end{tabular}

Abb. 6: Felderstruktur von Komplementierersätzen

Konjunktionale Einleiter von Argumentsätzen werden als Komplementierer bezeichnet. Sie sind syntaktische Köpfe. Der Rest des Nebensatzes, den sie einleiten, ist ihre Ergänzung.

Wird ein Komplementierersatz in Subjektfunktion nachgestellt, so wird im Vor- oder Mittelfeld des Matrixsatzes - je nach Matrixprädikat obligatorisch oder fakultativ - ein koindiziertes Korrelatpronomen, gewöhnlich es, eingefügt:

(14) $\mathrm{Es}_{\mathrm{i}}$ wundert mich, [dass sie dort gute Pizza backen $]_{\mathrm{i}}$.

(15) Stimmt $e_{i}$, [dass Otto umziehen will $]_{i}$ ?

Bei vielen Matrixprädikaten steht auch $\mathrm{zu}$ nachgestellten Komplementierersätzen in Objektfunktion fakultativ oder obligatorisch ein Korrelatpronomen (vgl. ZIFONUN et al. 1997: 1483ff.). Dieses muss immer im Mittelfeld stehen (vgl. WÖLLSTEIN-LEISTEN et al. 1997: 110f.). Für Objektsätze, die eine Akkusativleerstelle sättigen, steht gewöhnlich das Pronomen es wie in (16). Für Objektsätze, die eine präpositionale Leerstelle sättigen, stehen Pronominaladverbien, die aus der Proform $d a$ und der betreffenden Präposition zusammengesetzt sind, wie in (17):

(16) Anna bedauert es $\mathrm{s}_{\mathrm{i}}$, [dass Otto umzieht $]_{\mathrm{i}}$.

(17) Anna freut sich da $\mathrm{i}_{\mathrm{r}}$ rüber, [dass Otto umzieht $]_{\mathrm{i}}$.

Als Komplementierer kommt außer dass vor allem ob in Betracht:

Otto will wissen, ob Anna verheiratet ist.

Daneben kann auch wenn als Komplementierer verwendet werden (vgl. FABRICIUS-HANSEN 1980). Wenn-Argumentsätze zu Verben müssen sowohl in Subjekt- als auch in Objektfunktion mit einem Korrelatpronomen im Matrixsatz (gewöhnlich es) koindiziert sein: 
Blühdorn, H. - Syntaktische Nebensatzklassen im Deutschen

(19a) Es $s_{i}$ gefiel ihm, [wenn sie ihn Otto nannte $]_{i}$.

(19b) Er genoss es $s_{i}$, [wenn sie ihn Otto nannte $]_{i}$.

Wenn- und ob-Argumentsätze sind ebenso wie dass-Argumentsätze auf Leerstellen bestimmter Prädikate beschränkt.

Komplementierersätze können nicht nur $\mathrm{zu}$ verbalen, sondern vereinzelt auch $\mathrm{zu}$ adjektivischen Prädikaten als Argumente auftreten, z.B. zu würdig:

(20) Jeder einzelne Vermisste ist würdig, dass man seiner gedenkt.

Eine wichtige Rolle spielen sie ferner als Argumente zu Prädikaten, die durch $s o, z u$, (nicht) genug oder ähnliche Ausdrücke hinsichtlich ihres Grades bestimmt sind. Solche Gradprädikate können als Verbgruppen auftreten wie so (sehr) lachen in (21), arbeitet so verzweifelt in (23), regnete so (sehr) in (24) oder regnete (nicht) stark genug in (25) und (26), aber auch als Prädikative wie so betrunken in (22) oder als Attribute wie zu groß in (27). Gradprädikate mit genug verlangen dass-Sätze als Ergänzung; Gradprädikate mit $z u$ oder nicht genug verlangen zusätzlich die Vergleichspartikel als. Bei Prädikaten mit so stehen dass-Argumentsätze ohne Vergleichspartikel oder ob- bzw. wenn-Argumentsätze mit Vergleichspartikel. In aller Regel werden Argumentsätze zu Gradprädikaten ausgeklammert:

(21) Wir mussten so (sehr) lachen, dass der Tisch umfiel.

(22) Otto war so betrunken, dass er nicht mehr geradeaus gehen konnte.

(23) Otto arbeitet so verzweifelt, als ob er den Teufel im Nacken hätte.

(24) Es regnete so (sehr), als wenn der Himmel alle Schleusen geöffnet hätte.

(25) Es regnete stark genug, dass die Straße nass wurde.

(26) Es regnete nicht stark genug, als dass wir einen Schirm gebraucht hätten.

(27) Er hatte einen zu großen Appetit, als dass er satt geworden wäre.

Über den syntaktischen Status solcher Nebensätze besteht Uneinigkeit in den Grammatiken. Oft werden sie als konsekutive oder komparative Adverbialsätze eingestuft (vgl. DUDEN 2009: 1089f., 1100ff.). Dagegen spricht, dass sie ihrer Form nach (als dass-, ob- oder wennSätze mit oder ohne Vergleichspartikel) von Klassen von Prädikatsausdrücken gefordert werden und einen ergänzungstypischen Beitrag zur Satzbedeutung leisten. Sie sind weder frei 
Blühdorn, H. - Syntaktische Nebensatzklassen im Deutschen

zum Matrixsatz hinzufügbar noch ohne Weiteres weglassbar, d.h. sie verhalten sich in jeder Hinsicht wie Argumentsätze und in keiner wie Adverbialsätze.

Valenzunabhängig ist die Verwendung von dass-Sätzen bei der Satzspaltung. Wenn ein Adverbiale abgespalten wird - in Beispiel (28) kursiv gesetzt -, so erhält der Satzrest, der dann als Subjekt des Kopulaverbs sein fungiert, häufig die Form eines dass-Satzes:

Dass Otto zu spät kam, war heute.

Bei umgekehrter Linearisierung tritt zum Kopulaverb ein mit dem Subjektsatz koindiziertes Korrelatpronomen (gewöhnlich es):

(28a) Es $_{\mathrm{i}}$ war heute, [dass Otto zu spät kam $]_{i}$.

\subsection{V2-Sätze}

V2-Sätze in Argumentfunktion kommen besonders häufig bei Verben des Sagens und Denkens vor, vor allem als Objektsätze wie in (29), aber gelegentlich auch als Subjektsätze wie in (30) (vgl. ZIFONUN et al. 1997: 1453, 1458; AUER 1998):

(29) Otto glaubt, er sei Napoleon.

(30) Auf dem Zettel stand, Otto sei Napoleon.

\begin{tabular}{l|l|l|l|l} 
VF & LK & MF & RK & NF \\
\hline Otto & sei & Napoleon & &
\end{tabular}

Abb. 7: Felderstruktur von V2-Sätzen

Mit solchen Nebensätzen kann der Sprecher eine Äußerung oder einen Gedanken einer anderen Person wiedergeben. Dabei hat die Unterscheidung zwischen den Verbmodi Indikativ und Konjunktiv eine wichtige Funktion:

(31a) Otto sagt, Anna ist verheiratet.

(31b) Otto sagt, Anna sei verheiratet.

Pandaemonium, São Paulo, v. 16, n. 21, Jun/2013, p.149-189 www.fflch.usp.br/dllm/alemao/pandaemoniumgermanicum 
Blühdorn, H. - Syntaktische Nebensatzklassen im Deutschen

Durch die Wahl des Konjunktivs zeigt der wiedergebende Sprecher an, dass die Verantwortung für die wiedergegebene Aussage nicht bei ihm selbst, sondern bei der zitierten Person liegt (vgl. DIEWALD 1999: 182ff.). Die Wahl des Indikativs ist diesbezüglich neutral, das heißt, die Verantwortung für die Aussage liegt bei der zitierten Person, muss aber nicht auf diese beschränkt bleiben, sondern kann auch auf den wiedergebenden Sprecher übertragen werden. Gibt der Sprecher eine von ihm selbst gemachte Aussage wieder, so wird bevorzugt der Indikativ gewählt:

Ich sagte, Anna ist verheiratet.

Als V2-Argumentsätze im engeren Sinne können nur Aussagesätze verwendet werden. WFragesätze mit V2-Stellung sind in dieser Funktion ungrammatisch:

*Otto wollte wissen, wer sei der Halter dieses Hundes.

Auch V1-Frage- oder Aufforderungssätze können nicht als Argumentsätze im engeren Sinne verwendet werden:

(34) *Otto wollte wissen, sei das Annas Hund.

(35) *Otto forderte sie auf, hole sie ihm ein Bier.

Einschränkend muss man hinzufügen, dass bei der wörtlichen Redewiedergabe Sätze aller Art zugelassen sind:

(33a) Otto wollte wissen: „Wer ist der Halter dieses Hundes?“

(34a) Otto wollte wissen: „Ist das Annas Hund?“

(35a) Otto forderte sie auf: „Hol mir ein Bier.“

Als wörtliche Rede können außer Sätzen auch beliebige sonstige, sogar ungrammatische Ausdrücke wiedergegeben werden. Insofern sind wörtliche Redewiedergaben nicht zu den Nebensätzen im engeren Sinne zu rechnen. Im Schrifttext wird wörtliche Redewiedergabe durch Anführungsstriche oder andere Interpunktionsmittel gekennzeichnet. In gesprochenen Äußerungen kann sie durch prosodische Mittel markiert werden. Im konkreten Einzelfall ist 
Blühdorn, H. - Syntaktische Nebensatzklassen im Deutschen

die Unterscheidung zwischen wörtlicher Redewiedergabe und Redewiedergabe durch einen Nebensatz im engeren Sinne nicht immer eindeutig zu treffen.

Bei V2-Sätzen, die Kopulaverben ergänzen, kann zweifelhaft sein, ob sie als Subjekt oder als Prädikativ fungieren:

Die wichtigste Erkenntnis ist: Schalke kann noch gewinnen.

Die Leerstellen des Kopulaverbs sein sind hier mit einer Nominalgruppe und einem V2-Satz belegt. Auf den ersten Blick scheint sich eine Analyse anzubieten, die die Nominalgruppe als Subjekt und den V2-Satz als Prädikativ deutet. Bei genauerer Betrachtung erweist sich jedoch die umgekehrte Analyse als plausibler. Die Nominalgruppe die wichtigste Erkenntnis steht für eine Eigenschaft, die der im V2-Satz formulierten Aussage als Funktionsbeschreibung zugeordnet wird. Beschreibungen zuzuordnen ist die prototypische Aufgabe von Prädikativen. Anstelle der Nominalgruppe könnte in (36) auch ein prädikatives Adjektiv eingesetzt werden:

(36a) Klar ist: Schalke kann noch gewinnen.

Setzt man anstelle des V2-Satzes eine Nominalgruppe ein, so muss das Kopulaverb mit dieser kongruieren:

(36b) \{Erkenntnisse, die im Fußball gewonnen werden, beschränken sich oft auf die Faktizität von Sieg oder Niederlage.\}

Die wichtigste Erkenntnis sind (*ist) für uns die drei Punkte.

(36a/b) deuten übereinstimmend darauf hin, dass in (36) die Nominalgruppe als Prädikativ und der V2-Satz als Subjekt fungiert.

\subsection{Vergleichspartikelsätze}

Als Argumente zu Grad- und Vergleichsprädikaten können außer Komplementierersätzen auch VE-Sätze auftreten, die mit einer der Vergleichspartikeln wie, als oder je + Komparativ eingeleitet sind. Vergleichspartikelsätze werden in den Grammatiken üblicherweise nicht als 
Blühdorn, H. - Syntaktische Nebensatzklassen im Deutschen

eigene Satzart geführt. Meist werden sie als komparative bzw. proportionale Adverbialsätze eingestuft (vgl. DUDEN 2009: 1100ff.). Vergleichspartikelsätze haben aber, ebenso wie Komplementierersätze bei Gradprädikaten, keine der typischen Eigenschaften von Adverbialsätzen. Sie sind weder frei zum Matrixsatz hinzufügbar noch ohne Weiteres weglassbar. Sie werden ihrer grammatischen Form nach von Klassen von Prädikatsausdrücken selegiert. Somit müssen sie als Argumentsätze eingestuft werden. ${ }^{4}$

Wie-Sätze ergänzen Prädikate, die durch so modifiziert sind; als-Sätze ergänzen Prädikate im Komparativ oder Antezedentien wie anders oder insofern; je-Sätze ergänzen Prädikate im Komparativ, die durch desto modifiziert sind. Die ergänzten Prädikate können unterschiedliche Satzgliedfunktionen ausüben. Es können Verbgruppen sein wie mehr gearbeitet in (38), Prädikative wie desto dünner in (43), Adverbialia wie anders in (39), insofern in (40), desto begeisterter in (41) oder desto schneller in (42), oder Attribute wie so teuer in (37). Vergleichspartikelsätze werden typischerweise ausgeklammert wie in (37), (38), (39) und (41). Teilweise können sie auch Positionen im Vor- oder Mittelfeld des Matrixsatzes einnehmen wie in (40), (42) und (43), aber nur, wenn sie unmittelbar an die Konstituente angrenzen, die sie ergänzen. In Sätzen wie (42) steht der je-Satz zusammen mit dem Gradprädikat im Vorfeld:

Anna hat ein so teures Auto, wie es sich kaum jemand leisten kann.

Sie hat mehr gearbeitet, als sie musste.

Alles kam anders, als man gedacht hatte.

Insofern, als man ihr keine Vorschriften machte, war sie zufrieden. Sie wurde desto begeisterter gefördert, je größer ihr Fleiß war.

Je mehr sie unterstützt wurde, desto schneller stieg sie auf.

Die Luft wird, je höher man steigt, desto dünner.

Die syntaktische Struktur von Vergleichspartikelsätzen gleicht der von Komplementierersätzen. Die Vergleichspartikel nimmt die linke Klammerposition ein, das Vorfeld ist unbesetzt. $J e$-Sätze müssen einen Komparativ enthalten, der im Mittelfeld vorangestellt wird:

\footnotetext{
${ }^{4}$ In der Dudengrammatik (2009: 1030ff.) wird eine Einordnung als Relativsätze vorgeschlagen. Diese Alternative kann ich hier nicht im Detail diskutieren.
} 
Blühdorn, H. - Syntaktische Nebensatzklassen im Deutschen

\begin{tabular}{l|l|l|l|l} 
VF & LK & MF & RK & NF \\
\hline \multirow{2}{*}{ als } & es sich kaum jemand & leisten kann & \\
& je & höher man & musste & \\
& steigt &
\end{tabular}

Abb. 8: Felderstruktur von Vergleichspartikelsätzen

\subsection{V1-Sätze}

Als Argumente zu Gradprädikaten mit so können außer Komplementierersätzen und Vergleichspartikelsätzen auch V1-Sätze fungieren, denen die Vergleichspartikel als vorangestellt ist:

$$
\text { Anna war so betrunken, als wäre das Bier Wodka gewesen. }
$$

Im V1-Satz nimmt das Finitum die linke Klammerposition ein; das Vorfeld ist unbesetzt:

\begin{tabular}{l|l|l|l|l} 
VF & LK & MF & RK & NF \\
\hline & wäre & das Bier Wodka & gewesen &
\end{tabular}

Abb. 9: Felderstruktur von V1-Sätzen

In Sätzen, deren linke Klammerposition durch das Finitum besetzt ist, sind Subjunktoren nicht zugelassen (vgl. STERNEFELD 2008/2009: 322). Als kann in solchen Sätzen nicht als Subjunktor gedeutet werden. Es bleibt aber ein syntaktischer Kopf, kann also auch nicht im Vorfeld stehen, das für Phrasen reserviert ist. Somit muss der Nebensatz in (44) ein V1-Satz sein, der die Vergleichspartikel als ergänzt. Die Vergleichspartikel steht außerhalb des Nebensatzes. Deshalb wird sie im Felderschema in Abb. 9 nicht gezeigt. Die Vergleichspartikel mit ihrer Ergänzung (die Vergleichspartikelphrase) ergänzt ihrerseits das Gradprädikat.

Vergleichspartikeln erweisen sich als syntaktisch recht flexibel. Sie können die linke Klammerposition von VE-Sätzen (Vergleichspartikelsätzen) einnehmen, können aber auch 
Blühdorn, H. - Syntaktische Nebensatzklassen im Deutschen

Komplementierer- oder V1-Sätze als Komplement nehmen. Daneben können sie durch Ausdrücke zahlreicher weiterer Klassen (Nominalgruppen: als Lehrer, Adverbien: wie heute u.a.) ergänzt werden, die nicht Gegenstand dieses Aufsatzes sind.

\section{Prädikativsätze}

Nebensätze in der syntaktischen Funktion von Prädikativen (vgl. ZIFONUN et al. 1997: 1461; DUDEN 2009: 1027f.) sind in traditionellen Grammatiken kaum behandelt worden. Prädikative sättigen die zweite Leerstelle von Kopulaverben wie sein oder bleiben. In dieser Funktion können freie Relativsätze, Komplementierersätze und V1-Sätze verwendet werden. V2-Sätze scheinen nicht als Prädikative fungieren zu können (s.o. Abschnitt 2.4). Zumindest sind mir keine Beispiele bekannt, die eine entsprechende Analyse nahelegen.

\subsection{Freie Relativsätze}

(45) und (46) zeigen freie Relativsätze in Prädikativfunktion:

(45) Otto ist auf keinen Fall, was du ihm unterstellst.

(46) Otto bleibt, was er immer gewesen ist.

Auch in dieser Funktion können freie Relativsätze generalisierend oder spezifisch gelesen werden. In generalisierender Lesart besagt (45), dass jede beliebige Eigenschaft, die Otto unterstellt werden könnte, nicht auf ihn zutrifft. In spezifischer Lesart geht es um eine bestimmte Eigenschaft, die Otto aktuell von dem Adressaten unterstellt wird, an den der Sprecher sich wendet.

In sogenannten Pseudospaltsätzen (vgl. ZIFONUN et al. 1997: 231, 528f.; BLÜHDORN 2012a: 194ff.), in denen ein Argument nach rechts abgespalten wird, erhält der Satzrest die Form eines vorangestellten freien Relativsatzes in der Satzgliedfunktion eines Prädikativs. Das zeigen Beispiele wie (47) bis (49). Das finite Verb (sein) kongruiert mit dem abgespaltenen Argument, nicht mit dem freien Relativsatz. Das abgespaltene Argument 
Blühdorn, H. - Syntaktische Nebensatzklassen im Deutschen

fungiert als Subjekt. Das w-Relativum fungiert als Variable, die im Satzrest die grammatische Funktion anzeigt, in der das abgespaltene Argument zu interpretieren ist:

(47) Wer ${ }_{\mathrm{i}}$ hier wohnt, (*ist) sind [Otto und Anna $]_{\mathrm{i}}$.

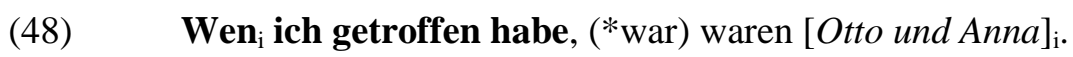

(49) Wer ${ }_{\mathrm{i}}$ hier für Ruhe sorgt und wem $_{\mathrm{i}}$ ich dafür dankbar bin, ist (*sind) $[\mathrm{Otto}]_{\mathrm{i}}$.

Wird das Argument nach links abgespalten, so erhält der Satzrest ebenfalls die Form eines Relativsatzes, der dann im Nachfeld steht. Er wird nicht mit einem w-, sondern mit einem dRelativum eingeleitet. Zum Kopulaverb tritt das Korrelatpronomen es, das mit dem Relativsatz als ganzem koindiziert ist:

(47a) [Otto und Anna $]_{\mathrm{j}}$ sind $\mathrm{es}_{\mathrm{i}}$ gewesen, $\left[\mathbf{d i e}_{\mathrm{j}} \text { hier wohnten }\right]_{\mathrm{i}}$.

(48a) $\mathrm{Es}_{\mathrm{i}}$ sind [Otto und Anna $]_{\mathrm{j}}$ gewesen, $\left[\mathbf{d i e}_{\mathrm{j}} \text { ich getroffen habe }\right]_{\mathrm{i}}$.

(49a) $\mathrm{Es}_{\mathrm{i}}$ ist Otto $_{\mathrm{j}}$ gewesen, [der $\mathrm{d}_{\mathrm{j}}$ hier für Ruhe sorgte und $\mathbf{d e m}_{\mathrm{j}}$ ich dafür dankbar war $]_{\mathrm{i}}$.

Relativsätze wie in (47a) bis (49a) werden in der Literatur gelegentlich als restriktive (notwendige) Attributsätze zum abgespaltenen Argument gedeutet (etwa in DUDEN 2009: 1035f.). Einer solchen Deutung ist nicht zuzustimmen. Es handelt sich um freie Relativsätze in Prädikativfunktion. Die Relativpronomina zeigen die grammatische Funktion an, in der die abgespaltene Konstituente zu interpretieren ist. Das Korrelatpronomen es steht für die Prädikativstelle des Matrixsatzes, die dem ausgeklammerten Relativsatz zukommt.

Vergleichen wir zur Verdeutlichung (50) mit (51):

(50) $\quad$ Wer ist [das auf dem Bild $]_{i}$ ? $\}$

es ist der /MANN der NElben uns wohnt ${ }^{5}$

$\mathrm{Es}_{\mathrm{i}}$ ist $[\text { der Mann }]_{\mathrm{j}}$, der ${ }_{\mathrm{j}}$ neben uns wohnt.

\footnotetext{
${ }^{5}$ Die Wortketten in (50) und (51) sind identisch, aber die Sätze unterscheiden sich in der Informationsstruktur, die durch die Intonation angezeigt wird. Ich schreibe Großbuchstaben für Akzentsilben sowie steigende und fallende Schrägstriche für steigende und fallende Tonbewegungen. In geschweiften Klammern steht verdeutlichendes Kontextmaterial.
} 
Blühdorn, H. - Syntaktische Nebensatzklassen im Deutschen

(51) \{wohnt die /FRAU neben euch?\}

$\{/ \mathrm{NEIN} \backslash-$ \} es ist der /MANN $\backslash$ der neben uns wohnt

$\mathrm{Es}_{\mathrm{i}}$ ist $[\operatorname{der} M a n n]_{\mathrm{j}},\left[\mathbf{d e r}_{\mathrm{j}} \text { neben uns wohnt }\right]_{\mathrm{i}}$.

(50) zeigt einen restriktiven Attributsatz zu Mann (s. dazu auch u. Abschnitt 5.2.1). Hier liegt kein Spaltsatz vor. Das Pronomen es nimmt das Prädikativ das auf dem Bild aus der vorausgegangenen Frage wieder auf. Der Relativsatz ist notwendig, um verstehen zu können, welcher Mann gemeint ist. (51) zeigt einen Spaltsatz. Das Subjekt der Mann wurde nach links abgespalten und steht in Kontrast zu die Frau aus der Frage. Das Pronomen es ist Korrelat zum Relativsatz. Dieser hat keinen Bezugsausdruck, ist also frei. Das Relativpronomen der fungiert als Variable für das abgespaltene Subjekt.

Auch freie d-Relativsätze können in Attributsätze zu d-Pronomina umgewandelt werden (s.o. Abschnitt 2.1):

(47b) [Otto und Anna $]_{\mathrm{i}}$ sind die $_{\mathrm{i}}\left(\mathbf{j e n i g e n )}\right.$ gewesen, $\mathbf{d i e}_{\mathrm{i}}$ hier wohnten.

Das d-Pronomen, das als Bezugsausdruck des Relativsatzes fungiert, erhält dann seine Position im Mittelfeld des Matrixsatzes. Das Korrelatpronomen es wird als Anzeiger für die grammatische Position des Relativsatzes überflüssig und fällt weg. Der Relativsatz kann ausgeklammert bleiben, wie in (47b). Er kann aber, bei geeigneter Informationsstruktur, auch direkt auf seinen Bezugsausdruck folgen. Im Vorfeld ist die Kontaktstellung generell präferiert:

(47c) [Otto und Anna $]_{\mathrm{i}}$ sind $\mathbf{d i e}_{\mathbf{i}}(\mathbf{j e n i g e n}), \mathbf{d i e}_{\mathrm{i}}$ hier wohnten, gewesen.

(47d) Die ${ }_{\mathrm{i}}\left(\mathbf{j e n i g e n )}, \mathbf{d i e}_{\mathrm{i}}\right.$ hier wohnten, sind $[\text { Otto und Anna }]_{\mathrm{i}}$ gewesen.

\subsection{Komplementierersätze}

Komplementierersätze in Prädikativfunktion müssen durch als oder wie eingeleitet sein:

(52) Otto sieht aus, als ob er Zahnschmerzen hätte.

(53) Minimal-invasive Chirurgie, das ist, wie wenn man mit Stäbchen isst. 
Blühdorn, H. - Syntaktische Nebensatzklassen im Deutschen

Ausdrücke wie als $o b$ und wie wenn werden in den Grammatiken häufig als zusammengesetzte Subjunktoren behandelt (z.B. in DUDEN 2009: 630). Meines Erachtens gibt es gute syntaktische Gründe, als und wie in Fällen wie (52) und (53) nicht als Bestandteile von Subjunktoren, sondern als vorangestellte Vergleichspartikeln zu deuten (s. Abschnitte 2.3, 2.5, 2.6 und 3.3). Als eigentliche Nebensatzeinleiter, die die linke Klammerposition einnehmen, bleiben unter einer solchen Analyse die Komplementierer $o b$ und wenn übrig:

\begin{tabular}{l|l|l|l|l} 
VF & LK & MF & RK & NF \\
\hline & ob & er Zahnschmerzen & hätte & \\
& wenn & man mit Stäbchen & isst &
\end{tabular}

Abb. 10: Felderanalyse für die Komplementierersätze aus (52) und (53)

\subsection{V1-Sätze}

Ein wichtiges Argument, das dagegen spricht, als und wie in prädikativen Vergleichssätzen als Bestandteile von Subjunktoren zu deuten, ist die Alternation von Komplementierersätzen mit V1-Sätzen:

\section{Otto sieht aus, als hätte er Zahnschmerzen.}

Minimal-invasive Chirurgie, das ist, als operierte man mit Essstäbchen.

Im V1-Satz nimmt das Finitum die linke Klammerposition ein; ein Subjunktor ist daher nicht zugelassen. In solchen Fällen gibt es keine Alternative dazu, als als Vergleichspartikel zu analysieren, die den V1-Satz als Ergänzung nimmt (s.o. Abschnitt 2.6):

\begin{tabular}{l|l|l|l|l} 
VF & LK & MF & RK & NF \\
\hline & hätte & er Zahnschmerzen & & \\
& operierte & man mit Essstäbchen & &
\end{tabular}

Abb. 11: Felderanalyse für die V1-Sätze aus (54) und (55) 
Blühdorn, H. - Syntaktische Nebensatzklassen im Deutschen

(52) und (53) unterscheiden sich von (54) und (55) nur darin, dass sie anstelle des V1-Satzes einen Komplementierersatz enthalten. In allen vier Fällen ist der prädikative Nebensatz Komplement einer Vergleichspartikel, was auch sonst bei Prädikativen häufig vorkommt (er war wie tot, er fühlte sich als Retter).

\section{Adverbialsätze}

Adverbialsätze sind satzförmig realisierte Adverbialia, also Lokal-, Temporal-, Konditional-, Kausal-, Konzessiv- usw. -angaben. Sie sind valenzunabhängig, können deshalb frei zum Matrixsatz hinzugefügt werden, soweit sie mit seiner Bedeutung verträglich sind, und können meist auch weggelassen werden, ohne die Grammatikalität des Satzes zu beeinträchtigen. Sie erweitern den Matrixsatz als syntaktische Adjunkte (vgl. BLÜHDORN/LOHNSTEIN 2012: 188ff.). In dieser Funktion kommen Sätze von drei Strukturtypen vor: freie Relativsätze, Subjunktorsätze und V1-Sätze.

\subsection{Freie Relativsätze}

Freie Relativsätze können z.B. als Orts- oder Zeitangaben wie in (56) und (57) fungieren:

(56) Wo sie hinschaute, gingen Menschen ihrer Arbeit nach.

(57) Wann immer du kommst, bist du willkommen.

Der freie Relativsatz in (56) kann - intonationsabhängig - spezifisch ('an dem bestimmten Ort, auf den der Blick der besprochenen Person traf') oder generalisierend gelesen werden ('an jedem beliebigen Ort, auf den ihr Blick treffen mochte'). Für den freien Relativsatz in (57) ist eine generalisierende Lesart zu wählen (' $z u$ jedem Zeitpunkt, zu dem du kommen magst').

Auch in anderen adverbialen Funktionen kommen freie Relativsätze vor:

(58) Mach es doch, wie du willst!

(59) Du kannst das Geld verwenden, wozu du es brauchst. 
Blühdorn, H. - Syntaktische Nebensatzklassen im Deutschen

In (58) fungiert der Relativsatz als Adverbiale der Art und Weise, in (59) als Adverbiale des Zwecks. In beiden Fällen ist sowohl eine spezifische ('auf die bestimmte vom Adressaten gewünschte Art und Weise', 'für den bestimmten vom Adressaten festgestellten Bedarf'), als auch eine generalisierende Lesart möglich ('auf eine beliebige Art und Weise', 'für jeden beliebigen Bedarf’).

\subsection{Subjunktorsätze}

Die prototypischen Beispiele für Adverbialsätze sind Subjunktorsätze:

(60) Als wir nach Hause kamen, war die Tür aufgebrochen.

(61) Da hier sowieso nichts mehr zu tun ist, können Sie gerne schon nach Hause gehen.

(62) Otto warf die Forelle in die Pfanne, während Anna Schubert hörte.

In (60) fungiert als nicht als Vergleichspartikel, sondern als Subjunktor. Subjunktorsätze haben die gleiche syntaktische Struktur wie Komplementierersätze. Der Subjunktor ist der Kopf, der Satzrest ist seine Ergänzung. Der Subjunktor nimmt die linke Klammerposition ein; das Vorfeld ist unbesetzt:

\begin{tabular}{l|l|l|l|l} 
VF & LK & MF & RK & NF \\
\hline & als & wir nach Hause & kamen & \\
& da & hier sowieso nichts mehr zu tun & ist \\
während & Anna Schubert & hörte &
\end{tabular}

Abb. 12: Felderstruktur von Subjunktorsätzen

Nicht alle Autoren unterscheiden zwischen Komplementierer- und Subjunktorsätzen, und diejenigen, die unterscheiden, tun es nicht auf einheitliche Weise. Meist wird die Grenze nach der syntaktischen Funktion gezogen: Komplementierersätze sind Argumentsätze, Subjunktorsätze sind Adverbialsätze (vgl. BLÜHDORN/LOHNSTEIN 2012: 184ff.). Ich folge dieser Konvention. Prädikativsätze habe ich analog zu Argumentsätzen behandelt. 
Blühdorn, H. - Syntaktische Nebensatzklassen im Deutschen

Subjunktorsätze können im Deutschen für die gesamte Bandbreite semantischer Adverbialfunktionen stehen, von Vergleich über räumliche und zeitliche Situierung bis hin zu den verschiedenen Arten von Konditionalität und Kausalität (vgl. BLÜHDORN 2010). Allerdings zeigen nicht alle Subjunktorsätze das gleiche grammatische Verhalten. Zum Beispiel können temporale als-Sätze wie in (60) im Skopus der Negation liegen, kausale daSätze wie in (61) dagegen nicht (vgl. BLÜHDORN 2012a: 381ff., 413ff.):

(60a) Die Tür war aufgebrochen, aber nicht als wir nach Hause kamen.

(61a) Sie können gerne schon nach Hause gehen, *aber nicht da hier sowieso nichts mehr zu tun ist.

Während-Sätze wie in (62) haben eine temporale und eine adversative Lesart. Wird der während-Satz jedoch negiert, bleibt nur die temporale Lesart möglich (vgl. ebd.: 259f.):

(62a) Otto warf die Forelle in die Pfanne, aber nicht während Anna Schubert hörte.

Temporale als- und während-Sätze können abgespalten werden, kausale da-Sätze und adversative während-Sätze nicht (vgl. BLÜHDORN 2012b: 200). Der Nebensatz in (62b) ist nur temporal, nicht adversativ lesbar:

(60b) Es war als wir nach Hause kamen, dass die Tür aufgebrochen war.

(61b) *Es ist da hier sowieso nichts mehr zu tun ist, dass Sie gerne schon nach Hause gehen können.

(62b) Es war während Anna Schubert hörte, dass Otto die Forelle in die Pfanne warf.

Es gibt noch zahlreiche weitere grammatische Unterschiede zwischen den beiden Gruppen von Adverbialsätzen (vgl. FREY 2011). HAEGEMAN (2002, 2003, 2006) bezeichnet Adverbialsätze, die sich so verhalten wie temporale als- und während-Sätze, als zentral, Adverbialsätze, die sich so verhalten wie kausale $d a$ - und adversative während-Sätze, als peripher.

Auch Subjunktorsätzen (vor allem konditionalen) kann zusätzlich eine Vergleichspartikel vorangestellt werden: 
Blühdorn, H. - Syntaktische Nebensatzklassen im Deutschen

(63) Wenn man die Mautstelle passiert, zieht man am Automaten einen Schein, wie wenn man ins Parkhaus fährt.

(64) Genieße dein Leben, als ob du morgen sterben müsstest.

Gegen die verbreitete Analyse von wie wenn und als ob als komplexe Subjunktoren spricht wiederum die Alternation mit V1-Sätzen (s.u. Abschnitt 4.3). Da der Nebensatz in (64) syntaktisch als Adverbiale fungiert, ist $o b$ hier nicht als Komplementierer, sondern als Subjunktor einzustufen.

\section{3 \1-Sätze}

Neben Adverbialsätzen mit VE-Stellung kommen sogenannte uneingeleitete Adverbialsätze mit V1-Stellung vor (vgl. REIS/WÖLLSTEIN 2010: 118ff.; BLÜHDORN 2012c: 305ff.; BLÜHDORN/LOHNSTEIN 2012: 212ff.):

(65) [Liegen Ihre Reiterfahrungen schon weit zurück $]_{\mathrm{i}},\left(\mathrm{dann}_{\mathrm{i}}\right)$ können Sie auch zunächst eine Longestunde nehmen.

(66) Er könnte einen richtig guten Roman schreiben, nähme er sich nicht vor, dabei auch furchtbar schlau zu sein.

(67) [Hat er auch keine direkte Bedeutung für unsere Gesundheit $]_{\mathrm{i}},\left(\mathrm{so}_{\mathrm{i}}\right)$ beeinträchtigt Haarausfall doch das persönliche Wohlbefinden.

(68) Dieser Regierung ist jedes Mittel recht, ist es auch noch so unsozial.

(69) [Hatten wir morgens noch leichten Schneefall gehabt $]_{i},\left(\mathrm{so}_{\mathrm{i}}\right) \mathrm{kam}$ mittags die Sonne hervor.

In V1-Sätzen nimmt das finite Verb die linke Klammerposition ein. Ein Subjunktor ist unzulässig:

\begin{tabular}{l|l|l|l|l}
$\mathbf{V F}$ & $\mathbf{L K}$ & $\mathbf{M F}$ & $\mathbf{R K}$ & $\mathbf{N F}$ \\
\hline & liegen & Ihre Reiterfahrungen schon weit & zurück & \\
& hatten & wir morgens noch leichten Schneefall & gehabt &
\end{tabular}

Abb. 13: Felderstruktur von V1-Sätzen 
Blühdorn, H. - Syntaktische Nebensatzklassen im Deutschen

In vielen Darstellungen werden nur uneingeleitete Konditionalsätze wie in (65) und (66) erwähnt. V1-Adverbialsätze sind aber auch konzessiv wie in (67) und (68) und adversativ wie in (69) verwendbar (vgl. Engel 1996; Zifonun et al. 1997). Konditionale V1-Sätze können bedeutungserhaltend durch wenn-Sätze, konzessive durch auch wenn-Sätze, adversative durch während-Sätze - jeweils mit VE-Stellung - ersetzt werden. Konditionale und konzessive V1Sätze können dem Matrixsatz voran- oder nachgestellt werden. Bei Voranstellung nehmen sie das Vorfeld des Matrixsatzes ein. Sie können mit einem Resumptivum wie dann oder so koindiziert sein, das mit ihnen zusammen das Vorfeld einnimmt. Adversative V1-Sätze werden dem Matrixsatz immer vorangestellt.

Unter den Testverfahren wie Negation und Abspaltung (s.o. Abschnitt 4.2) erweisen sich V1-Adverbialsätze als syntaktisch peripher:

(65a) *Es ist liegen Ihre Reiterfahrungen schon weit zurück, dass Sie zunächst eine Longestunde nehmen können.

(67a) *Es ist hat er auch keine direkte Bedeutung für unsere Gesundheit, dass Haarausfall doch das persönliche Wohlbefinden beeinträchtigt.

(68a) Dieser Regierung ist jedes Mittel recht, *aber nicht ist es auch noch so unsozial.

Konditionalen adverbialen V1-Sätzen kann die Vergleichspartikel als vorangestellt werden:

Man zieht am Automaten einen Schein, als führe man ins Parkhaus.

\section{Attributsätze}

Attributsätze sind satzförmig realisierte Erweiterungen zu Satzgliedern, insbesondere zu Nominalgruppen. Als Beispiele werden in Grammatiken und Lehrbüchern überwiegend gebundene Relativsätze mit VE-Stellung angeführt. Daneben kommen aber auch freie Relativsätze, gebundene Relativsätze mit V2-Stellung, indirekte w-Fragesätze, Komplementierersätze, Subjunktorsätze und V1-Sätze für diese Funktion in Frage. 
Blühdorn, H. - Syntaktische Nebensatzklassen im Deutschen

\subsection{Freie Relativsätze}

Nur selten wird darauf hingewiesen, dass freie Relativsätze in Attributfunktion auftreten können. Solche Attributsätze müssen immer von einem Resumptivum gefolgt werden, das mit dem Relativsatz koindiziert ist. Ein Beispiel ist das Sprichwort in (71a), das durch (71b) in modernes Standarddeutsch übersetzt werden kann:

(71a) [Wes Brot ich ess $]_{i}$, des ${ }_{i}$ Lied ich sing.

(71b) [Wessen Brot ich esse $]_{i}$, dessen ${ }_{i}$ Lied singe ich.

Dass der Relativsatz in (71b) frei ist, erweist sich an der Möglichkeit, ihm ein d-Pronomen als Bezugsausdruck voranzustellen (s.o. Abschnitt 2.1):

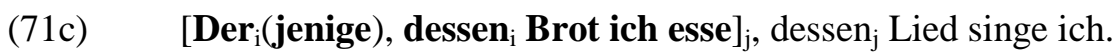

In (71b) ist das Resumptivum dessen mit dem freien Relativsatz als ganzem koindiziert. Relativsatz und Resumptivum fungieren gemeinsam als Genitivattribut zu dem Substantiv Lied. Der Relativsatz könnte aber nicht allein, ohne Resumptivum, die syntaktische Stelle des Attributs einnehmen:

(71d) $\quad *$ Wessen Brot ich esse Lied singe ich.

\subsection{Gebundene Relativsätze}

\subsubsection{VE-Relativsätze}

Relativsätze mit Bezugsausdruck sind typischerweise VE-Sätze. Bezugsausdruck kann ein Argument wie in (72), ein Prädikativ wie in (73), ein Adverbiale wie in (74) oder ein Attribut wie in (75) sein. Das Relativum ist mit dem Bezugsausdruck koindiziert:

(72) Wir vermissen [den Studenten $]_{\mathrm{i}}$, der $_{\mathrm{i}}$ heute vortragen soll.

(73) Eine Säge ist [ein Werkzeug $]_{i}$, mit $_{\text {dem }}$ man vorsichtig sein muss. 
Blühdorn, H. - Syntaktische Nebensatzklassen im Deutschen

(74) Gerade heute ${ }_{i}, \mathbf{w o}_{i}$ so schönes Wetter ist, müssen wir arbeiten.

(75) Das Referat [des Studenten $]_{i}$, der $_{i}$ heute vorgetragen hat, war beeindruckend.

Der Relativsatz fungiert syntaktisch als Attribut zu seinem Bezugsausdruck. Er muss in der Linearstruktur des Satzes aber nicht unbedingt direkt an den Bezugsausdruck angrenzen. Wir haben schon in den Abschnitten 2.1 und 3.1 gesehen, dass gebundene Relativsätze ausgeklammert werden können:

(72a) Wir haben [den Studenten $]_{\mathrm{i}}$ vermisst, der $_{\mathrm{i}}$ heute vortragen sollte.

(74a) Gerade heute $e_{\mathrm{i}}$ müssen wir arbeiten, wo $\mathbf{w}_{\mathrm{i}}$ so schönes Wetter ist.

Allgemein bekannt ist die Unterscheidung zwischen restriktiven und appositiven Relativsätzen (vgl. LEHMANN 1984: 261ff.; BLÜHDORN 2007: 294ff.). Restriktive Relativsätze sind nach verbreiteter Darstellung für die Interpretation ihres Bezugsausdrucks (und damit des Matrixsatzes) unentbehrlich. Wenn man sie weglässt, wird der Restsatz unverständlich. Hinter dieser Intuition steht die formale Eigenschaft, dass restriktive Relativsätze als Bestandteile von Beschreibungsausdrücken fungieren, mit denen keine eigene Aussage gemacht wird (vgl. BLÜHDORN 2007: 304ff.). Der Relativausdruck, der sie einleitet, ist eine rein formale Variable. Die Relativsätze in (72), (73) und (75) sind bevorzugt in diesem Sinne zu lesen.

Appositive Relativsätze wie in (74) sind dagegen nach landläufiger Darstellung für die Interpretation des Bezugsausdrucks (und damit des Matrixsatzes) entbehrlich. Wenn man sie weglässt, kann der Restsatz trotzdem noch angemessen verstanden werden. Dahinter steht die formale Eigenschaft, dass appositive Relativsätze eigene, zusätzliche Aussagen machen, die neben die Aussage des Restsatzes treten. Der Relativausdruck, der einen appositiven Relativsatz einleitet, ist ein referentieller Ausdruck.

Es ist eine viel diskutierte Frage, ob die Unterscheidung zwischen restriktiven und appositiven Relativsätzen in der Syntax wurzelt oder ob es sich um eine rein semantische Unterscheidung handelt (vgl. LEHMANN 1984: 261ff.; HOLLER 2005: 26, 68ff.; BLÜHDORN 2007: 296f.). Wurzelte die Unterscheidung in der Syntax, so wäre anzunehmen, dass restriktive Relativsätze syntaktisch tiefer eingebettet sind als appositive. Viele Autoren stellen beispielsweise restriktive Relativsätze mit nominalem Bezugsausdruck als Attribute zum Substantiv dar, appositive dagegen als Attribute zur ganzen Nominal- bzw. 
Blühdorn, H. - Syntaktische Nebensatzklassen im Deutschen

Determinansphrase. Solche syntaktischen Ableitungsversuche stoßen allerdings auf Schwierigkeiten, z.B. bei restriktiven Relativsätzen, die eine Koordination als Bezugsausdruck ${ }^{6}$ haben:

(76) [Die Bewerberin und der Bewerber $]_{i}$, die ${ }_{i}$ ihre Unterlagen zu spät eingereicht haben, werden nicht berücksichtigt.

Der Relativsatz in (76) ist bevorzugt restriktiv zu lesen. Lässt man ihn weg, so ist möglicherweise nicht mehr klar, welche Bewerber gemeint sind. Sein Relativpronomen steht im Plural. In seinem Bezugsausdruck gibt es aber kein Substantiv im Plural. Als Plural kann nur die Koordination als ganze gedeutet werden. Somit muss ein solcher Relativsatz Attribut zur gesamten Koordination sein, auch dann, wenn er restriktiv gelesen wird. Eine Deutung als Attribut zu einem der Substantive kommt aus formalen Gründen nicht in Frage. Fälle wie (76) sprechen stark dafür, Relativsätze generell als Attribute zum vollständigen Bezugsausdruck $\mathrm{zu}$ behandeln und nur semantisch zwischen restriktiver und appositiver Lesart zu unterscheiden.

Gebundene Relativsätze werden typischerweise mit einem d-Pronomen eingeleitet, freie mit einem w-Pronomen. In Abschnitt 3.1 haben wir gesehen, dass in besonderen Fällen auch freie d-Relativsätze vorkommen (vgl. dazu LEHMANN 1984: 293ff.). Ebenso gibt es gebundene w-Relativsätze, z.B. solche, bei denen als Bezugsausdruck ein d-Pronomen im Neutrum fungiert:

(77) Otto isst das $\mathbf{d}_{\mathrm{i}}, \mathbf{w a s}_{\mathrm{i}}$ auf seinem Teller liegt.

\subsubsection{V2-Relativsätze}

Eine Klasse von Nebensätzen, die vor allem in informeller und mündlicher Rede gebräuchlich ist und erst in jüngerer Zeit die Aufmerksamkeit von Grammatikern gefunden hat, sind Attributsätze mit V2-Stellung wie in den folgenden Beispielen:

\footnotetext{
${ }^{6}$ Den Hinweis darauf verdanke ich Joachim Jacobs (persönliche Mitteilung).
} 
Blühdorn, H. - Syntaktische Nebensatzklassen im Deutschen

(78) Das Buch hat [eine Seite $]_{i}$, die $_{i}$ ist ganz schwarz.

(79) Vulkanier sind Lebewesen ${ }_{i}$, die ${ }_{i}$ gibt's gar nicht wirklich.

Die Nebensätze in (78) und (79) sind an Bezugsausdrücke gebunden und restriktiv zu lesen. Sie haben rein beschreibende Funktion; ihre pronominalen Einleitungsausdrücke sind formale Variable. GÄRTNER (2001) rechnet solche V2-Nebensätze zu den Relativsätzen. Sie können ein Argument wie in (78) oder ein Prädikativ wie in (79) als Bezugsausdruck nehmen.

Baut man den Bezugsausdruck eines solchen V2-Satzes aus, sodass er allein als hinreichende Beschreibung, der V2-Satz als zusätzliche Aussage und sein einleitendes Pronomen als anaphorischer referentieller Ausdruck verstanden werden kann, so entfällt die Grundlage für die syntaktische Deutung des V2-Satzes als Attribut und Relativsatz:

(78a) Das Buch hat [eine komische Seite $]_{i}$, die $_{i}$ ist ganz schwarz.

(79a) Vulkanier sind [Science-Fiction-Lebewesen $]_{i}$, die $e_{i}$ gibt's gar nicht wirklich.

V2-Sätze wie in (78a) und (79a) werden nicht als appositiv, sondern als weiterführend (s.u. Kapitel 6) oder als selbständige neue Sätze gelesen (vgl. BLÜHDORN/LOHNSTEIN 2012: 220).

\subsection{Indirekte w-Fragesätze}

Relativsätze sind als Attribute stets valenzunabhängig, d.h. es gibt keine Bezugsausdrücke, die Relativsätze als syntaktische Ergänzung verlangen. Es gibt aber nominale Bezugsausdrücke, die Nebensätze anderer Klassen als Ergänzung verlangen. So können Substantive, deren Bedeutung auf eine zu schließende Wissenslücke hinweist, durch indirekte w-Fragesätze ergänzt werden. Meist sind solche Substantive deverbale oder deadjektivische Ableitungen:

(80) Die Frage, wer das Auto gestohlen hat, ist immer noch ungeklärt.

(81) Die Ungewissheit, wer der nächste sein würde, nagte an unseren Nerven. 
Blühdorn, H. - Syntaktische Nebensatzklassen im Deutschen

\subsection{Komplementierersätze}

Auch Komplementierersätze mit dass oder $o b$ können als valenzabhängige Attribute auftreten, vornehmlich zu deverbalen und deadjektivischen Ableitungen, die auf Konzepte des Sagens und Denkens bezogen sind:

(82) Es besteht noch Hoffnung, dass der Patient wieder gesund wird.

(83) Liebe ist die Gewissheit, dass nichts Besseres nachkommt.

(84) Alle hatten Zweifel, ob die Maßnahmen ausreichen würden.

\subsection{Subjunktorsätze}

Subjunktorsätze wie in (85) und (86) können als valenzunabhängige Attribute fungieren. (87) zeigt einen attributiven Subjunktorsatz, der zusätzlich durch eine Vergleichspartikel eingeleitet ist:

(85) Eine Ablehnung, weil das Geld fehlt, wäre eine Enttäuschung.

(86) Der Jubel, nachdem Schalke gewonnen hatte, war grenzenlos.

(87) Man hörte ein Geräusch, wie wenn einer versucht, kein Geräusch zu machen.

\subsection{V1-Sätze}

Auch durch die Vergleichspartikel als eingeleitete V1-Sätze können als valenzunabhängige Attribute fungieren:

(88) Dann folgte ein Blitz, als wäre ein Gaswerk in die Luft geflogen. 
Blühdorn, H. - Syntaktische Nebensatzklassen im Deutschen

\section{Desintegrierte und weiterführende Sätze}

Ich gehe noch kurz auf einige Satzarten ein, die bei oberflächlicher Betrachtung wie Nebensätze aussehen und ihnen in vielen Grammatiken auch zugerechnet werden (vgl. z.B. ENGEL 1996: 286, 294f.). Sie sind ihrem Bezugssatz meist nachgestellt, können nicht dessen Vorfeld einnehmen, teilweise aber vor seinem Vorfeld stehen. Im Mittelfeld des Bezugssatzes kommen sie allenfalls als Parenthesen vor. Sie sind keine Konstituenten des Bezugssatzes im engeren Sinne, sondern nur locker an ihn angeheftet (vgl. ZIFONUN et al. 1997: 2323ff.; HOLLER 2005; 2007). Auch pragmatisch sind sie als selbständig zu betrachten, da sie zur Ausführung eigener Sprechakte dienen. Sowohl in Schrifttexten als auch in der mündlichen Rede werden solche Sätze meist deutlich von ihrem Bezugssatz abgegrenzt: graphisch durch Interpunktionszeichen wie Doppelpunkte oder Gedankenstriche, intonatorisch durch Pausen oder durch selbständige Phrasierung.

Sätze, die wahlweise dem Vorfeld des Bezugssatzes vorangestellt oder dem Bezugssatz nachgestellt werden können, bezeichne ich als desintegriert, solche, die nur nachgestellt werden können, bezeichne ich als weiterführend.

Desintegrierte und weiterführende Sätze können als dritte Kategorie zwischen Nebensätzen und Hauptsätzen eingestuft werden. Sätze aller Strukturtypen können auf diese Weise verwendet werden. Ich gehe auf desintegrierte indirekte w-Fragesätze, weiterführende Relativsätze, desintegrierte und weiterführende Subjunktorsätze sowie desintegrierte und weiterführende V1-Sätze ein. Dass V2-Sätze weiterführend angeschlossen werden können, zeigen Beispiele wie (78a) und (79a) in Abschnitt 5.2.2. Der Übergang zu gewöhnlichen Nachfolgesätzen ist fließend.

\subsection{Desintegrierte indirekte w-Fragesätze}

Indirekte w-Fragesätze können verwendet werden, um Sätze oder Gruppen von Sätzen (bzw. die mit ihnen ausgeführten Sprechakte) pragmatisch $\mathrm{zu}$ kontextualisieren und $\mathrm{zu}$ kommentieren: 
Blühdorn, H. - Syntaktische Nebensatzklassen im Deutschen

(89) [Wir haben es mit einem Schwellenland zu tun $]_{i}$, was auch immer das $_{i}$ bedeuten mag.

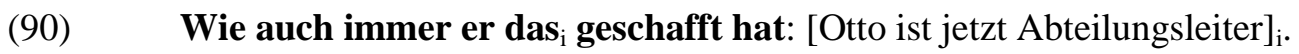

(91) Wichtig ist eine individuell passende Finanzierung - warum auch immer man sich zum Bauen entschließt.

Sätze dieser Art können ihrem Bezugssatz nachgestellt wie in (89) und (91) oder aber vorangestellt werden wie in (90). In beiden Fällen stehen sie syntaktisch außerhalb des Bezugssatzes, bei Voranstellung also vor dessen Vorfeld. Das einleitende Interrogativum ist nicht mit dem Bezugssatz oder einer seiner Konstituenten koindiziert. Eine referentielle Verknüpfung mit dem Bezugssatz kann durch ein d-Pronomen im Mittelfeld erfolgen wie in (89) und (90); sie kann aber auch fehlen wie in (91).

\subsection{Weiterführende Relativsätze}

Viele weiterführende Sätze haben die Struktur von Relativsätzen mit VE-Stellung. Sie können mit einem d- oder w-Relativum eingeleitet sein und Konstituenten oder Sätze als Bezugsausdruck nehmen (vgl. HOLLER 2005; 2007).

\subsubsection{Konstituentenbezogene Relativsätze}

Konstituentenbezogene weiterführende Relativsätze ähneln appositiven Relativsätzen, sind aber lockerer als diese angeschlossen:

(92) In der achten Klasse hatte sie [einen alten mürrischen Lateinlehrer $]_{i}-$ der $_{\mathrm{i}}$ ihr allerdings viel später noch sehr nützlich werden sollte.

In gewisser Weise sind sie komplementär zu V2-Relativsätzen. Prototypische Relativsätze haben VE-Stellung und können restriktiv gelesen werden. V2-Relativsätze müssen restriktiv gelesen werden, haben aber keine VE-Stellung. Weiterführende Relativsätze haben VEStellung, können aber nicht restriktiv gelesen werden. 
Blühdorn, H. - Syntaktische Nebensatzklassen im Deutschen

Wo genau die Grenze zwischen appositiven und weiterführenden konstituentenbezogenen Relativsätzen verläuft, ist nicht immer klar (vgl. HoLLER 2007: 260ff.). Dazu muss noch weiter geforscht werden.

\subsubsection{Satzbezogene Relativsätze}

Häufig ist der Relativausdruck von weiterführenden Relativsätzen nicht mit einer Konstituente, sondern mit einem vorausgehenden Satz (oder mit einer vorausgehenden Sequenz von Sätzen) koindiziert (vgl. DUDEN 2009: 1037):

(93) [Otto ist in allen Prüfungen durchgefallen $]_{i}$, was w $_{\mathrm{i}}$ letztlich niemanden überrascht hat.

(94) [Otto versuchte sofort, ein Gespräch anzufangen $]_{i}$, woffür ich ihm unendlich dankbar war.

(95) [In den meisten Bereichen sind besondere Fachkenntnisse unerlässlich $]_{i}$, wes:halb firmeninterne Schulungen angeboten werden.

Die Relativausdrücke in solchen Beispielen sind Komplex-Anaphern im Sinne von ScHWARZFRIESEL et al. (2004). Innerhalb des Relativsatzes kann der Relativausdruck als Argument wie in (93) und (94) oder als Adverbiale wie in (95) fungieren.

Relativadverbien wie weshalb in (95) enthalten eine pronominale Komponente (hier wes-), die mit ihrem Bezugsausdruck koindiziert ist, und eine präpositionale Komponente (hier -halb), die für eine semantische Verknüpfungsrelation steht (vgl. BLÜHDORN 2012b: 198ff., 207f.). Wie alle Relativausdrücke stehen Relativadverbien im Vorfeld:

\begin{tabular}{l|l|l|l|l} 
VF & LK & MF & RK & NF \\
\hline weshalb & & firmeninterne Schulungen & angeboten werden &
\end{tabular}

Abb. 14: Felderstruktur von Relativsätzen mit Relativadverb

Interessant sind Fälle, in denen ein weiterführender Relativsatz an einen Nebensatz als Bezugsausdruck anschließt, der seinerseits in einen übergeordneten Matrixsatz integriert ist: 
Blühdorn, H. - Syntaktische Nebensatzklassen im Deutschen

(96) Klar ist zur Zeit nur, [dass [drei der Männer das Feuer eröffneten $]_{i}$, wo $\mathbf{w}_{\mathrm{i}}$ rauf die Gäste in Panik gerieten].

Bezugssatz und weiterführender Relativsatz nehmen hier gemeinsam die Subjektstelle des Prädikats klar sein ein. Die gemeinsame Einbettung erleichtert es, Bezugssatz und Relativsatz als syntaktisch verbunden zu lesen. In solchen Kontexten scheint es sinnvoll, von appositiven satzbezogenen Relativsätzen zu sprechen (vgl. BLÜHDORN/LOHNSTEIN 2012: 198ff.)

\subsection{Desintegrierte und weiterführende Adverbialsätze}

Desintegrierte und weiterführende Sätze weisen oft Strukturen auf, die für Adverbialsätze kennzeichnend sind. Es handelt sich um subjunktoreingeleitete VE-Sätze und um V1-Sätze.

\subsubsection{Subjunktorsätze}

Subjunktorsätze stehen manchmal desintegriert vor dem Vorfeld ihres Bezugssatzes:

\{Ein Dispositionskredit kommt für mich nicht in Frage, \} und wenn ich es mal so sagen darf, die Idee mit der Kreditkarte ist Blödsinn.

(98) \{Otto hätte es eigentlich besser wissen müssen, \} aber obgleich er dagegen war - er hat mitgemacht.

BLÜHDORN (2012b: 201) bezeichnet die Position, die von vorangestellten desintegrierten Sätzen eingenommen wird, als linke externe Adjunktposition (LEA). Sie liegt zwischen einem möglichen Konjunktor - und in (97), aber in (98) - und dem Vorfeld des Bezugssatzes. Desintegrierte vorangestellte Subjunktorsätze kontextualisieren, ähnlich wie desintegrierte indirekte w-Fragesätze, den Sprechakt, der mit dem Bezugssatz ausgeführt wird.

Desintegrierte konditionale Subjunktorsätze können zusätzlich mit der Vergleichspartikel als eingeleitet werden:

(99) Als ob wir nicht schon vor Monaten davor gewarnt hätten - die Betriebsleitung hat jetzt Kurzarbeit angekündigt. 
Blühdorn, H. - Syntaktische Nebensatzklassen im Deutschen

Da es sich um einen Adverbialsatz handelt, ist $o b$ hier nicht als Komplementierer, sondern als Subjunktor einzustufen. Der Subjunktorsatz ergänzt die Vergleichspartikel.

Manche Subjunktorsätze werden ihrem Bezugssatz generell nachgestellt. Dazu gehören z.B. kausale zumal-Sätze:

(100) Die Risiken der Atomkraft werden heute verstärkt wahrgenommen, zumal sie ja bis vor kurzem noch völlig unbekannt waren.

(100a) *Zumal die Risiken der Atomkraft ja bis vor kurzem noch völlig unbekannt waren, werden sie heute verstärkt wahrgenommen.

PASCH et al. (2003: 418ff.) bezeichnen die Einleiter solcher Sätze als Postponierer.

Gewöhnliche Subjunktoren erlauben es, den Nebensatz, den sie einleiten, ins Vorfeld des Matrixsatzes zu stellen oder ihn dem Matrixsatz nachzustellen. Nachgestellte Subjunktorsätze können aber Interpretationen zulassen, die für Subjunktorsätze im Vorfeld ausgeschlossen sind. In (101) beschreibt der vorangestellte als-Satz einen zeitlichen Kontext, in dem es der Fall war, dass der Sprecher an der Haustür stand. In (101a) kann der nachgestellte als-Satz ebenso gelesen werden:

(101) Als das Gewitter einsetzte, stand ich gerade an der Haustür.

(101a) Ich stand gerade an der Haustür, als das Gewitter einsetzte.

Dagegen berichtet der nachgestellte als-Satz in (102) (aus Zifonun et al. 1997: 2326) von einem nächsten Schritt in einer Ereignissequenz. Zifonun et al. zufolge (ebd.: 2325f.) liefert in solchen Beispielen der Bezugssatz den zeitlichen Kontext für den nachgestellten als-Satz, d.h. in (102) wird das Stehen des Sprechers an der Haustür als Kontext für das Einsetzen des Gewitters gedeutet. Die Kontextualisierungsrelation verläuft also genau umgekehrt zu (101a):

(102) Ich stand gerade an der Haustür, als plötzlich ein schreckliches Gewitter einsetzte.

Für als-Sätze im Vorfeld sind solche Lesarten ausgeschlossen. (102a) muss analog zu (101) so gedeutet werden, dass das Einsetzen des Gewitters den zeitlichen Kontext für das Stehen des Sprechers an der Haustür bildet: 
Blühdorn, H. - Syntaktische Nebensatzklassen im Deutschen

(102a) Als plötzlich ein schreckliches Gewitter einsetzte, stand ich gerade an der Haustür.

Postponierersätze wie in (100), die nicht das Vorfeld einnehmen können, und nachgestellte Subjunktorsätze wie in (102), deren Lesart bei Vorfeldstellung nicht erhalten bleibt, sind weiterführend. BLÜHDORN (2012b: 204) bezeichnet die syntaktische Position, die sie relativ zum Matrixsatz einnehmen, als rechte externe Adjunktposition (REA). Die syntaktische Abgrenzung zwischen dem Nachfeld, das gewöhnliche Subjunktorsätze wie in (101a) einnehmen, und der REA, die weiterführenden Sätzen vorbehalten ist, kann im Einzelfall schwierig sein. Dazu muss noch Forschungsarbeit geleistet werden.

\subsubsection{V1-Sätze}

Desintegrierte Konditionalsätze, die die Vergleichspartikel als ergänzen, können V1-Stellung aufweisen. Sie können dem Bezugssatz voran- oder nachgestellt werden:

(103) Die Haltung ungewöhnlicher Wildtiere kommt in Mode - als hätten wir sonst keine Sorgen.

Weiterführend sind kausale V1-Sätze mit der Partikel doch im Mittelfeld (vgl. ENGEL 1996: 269). Im Unterschied zu konditionalen, konzessiven und adversativen V1-Sätzen (s.o. Abschnitt 4.3) müssen sie dem Restsatz stets nachgestellt werden:

(104) Wir waren alle sehr traurig, mussten wir doch unsere Freunde zurücklassen.

(104a) $*$ [Mussten wir doch unsere Freunde zurücklassen $]_{i},\left(\mathrm{so}_{\mathrm{i}}\right)$ waren wir alle sehr traurig.

Kausale V1-Sätze können bedeutungserhaltend durch kausale wo- oder $d a$-Sätze mit VEStellung substituiert werden:

(104b) Wir waren alle sehr traurig, wo/da wir (doch) unsere Freunde zurücklassen mussten. 
Blühdorn, H. - Syntaktische Nebensatzklassen im Deutschen

\section{Fazit}

Die Nebensätze des Deutschen können nach ihrer syntaktischen Funktion im Matrixsatz in Argument-, Prädikativ-, Adverbial- und Attributsätze unterteilt werden. Sie sind von desintegrierten und weiterführenden Sätzen zu unterscheiden, die nebensatzähnlich aussehen, aber keine Konstituenten ihres Bezugssatzes sind.

Nach ihrer syntaktischen Struktur sind Nebensätze mit VE-Stellung, V1-Stellung und V2-Stellung zu unterscheiden. Die VE-Sätze zerfallen weiter in solche mit besetztem Vorfeld und leerer linker Klammerposition (Relativsätze und indirekte w-Fragesätze) und solche mit leerem Vorfeld und besetzter linker Klammerposition (Komplementierer-, Subjunktor- und Vergleichspartikelsätze).

Abbildung 15 zeigt die Verteilung der Strukturtypen auf die Funktionstypen. In manchen Fällen muss dem Nebensatz eine Vergleichspartikel vorangestellt werden angezeigt durch die nicht-eingeklammerte Abkürzung Vpart -; in anderen ist die Voranstellung einer Vergleichspartikel möglich - angezeigt durch (Vpart):

\begin{tabular}{l|c|c|c|c} 
& \multicolumn{2}{|c|}{ VE-Sätze mit } & V1-Sätze & V2-Sätze \\
& besetztem Vorfeld & unbesetztem Vorfeld & \\
\hline Argumentsätze & + & + (Vpart $)$ & + Vpart & + \\
Prädikativsätze & + & + Vpart & + Vpart & $-?$ \\
Adverbialsätze & + & $+($ Vpart $)$ & $+($ Vpart $)$ & - \\
Attributsätze & + & $+($ Vpart $)$ & + Vpart & + \\
\hline desintegr. und & + & $+($ Vpart $)$ & $+($ Vpart $)$ & $(+)$ \\
weiterf. Sätze & + & &
\end{tabular}

Abb. 15: Funktions- und Strukturtypen deutscher Nebensätze

In Argument- und Attributfunktion können Sätze aller Strukturtypen auftreten, V1-Sätze allerdings nur mit vorangestellter Vergleichspartikel. Auch in Prädikativfunktion müssen V1Sätze durch eine Vergleichspartikel eingeleitet sein. Ohne Vergleichspartikel kommen V1- 
Blühdorn, H. - Syntaktische Nebensatzklassen im Deutschen

Nebensätze nur als Adverbialia vor. VE-Sätze kommen in allen Satzgliedfunktionen vor. Als Prädikative müssen sie bei unbesetztem Vorfeld durch eine Vergleichspartikel eingeleitet sein. V2-Sätze können nicht als Adverbialia fungieren. Ob sie als Prädikative vorkommen, ist fraglich. Desintegriert und weiterführend können Sätze aller Strukturtypen verwendet werden. Der Übergang zwischen weiterführenden V2-Sätzen und gewöhnlichen Nachfolgesätzen ist fließend.

Die Unterscheidung zwischen freien w-Relativsätzen und indirekten w-Fragesätzen scheint vornehmlich semantisch begründet zu sein. Ein syntaktischer Unterschied zeigt sich nur bei der Selektion durch Valenzträger und bei der Auswahl vorausgehender Proformen zu Subjektsätzen (es für indirekte Fragesätze vs. d-Pronomina für freie Relativsätze). Bei den Subjunktorsätzen kann nach syntaktischen Kriterien zwischen zentraler und peripherer Adjunktion unterschieden werden. Bei den Relativsätzen lässt sich die Unterscheidung zwischen restriktiver und appositiver Lesart wahrscheinlich nur semantisch, nicht syntaktisch explizieren. Bei den freien Relativsätzen hat die semantische Unterscheidung zwischen generalisierenden und spezifischen Lesarten mit Sicherheit keine syntaktische Grundlage.

Als Vertiefungsaspekte wurden nebensatzspezifische Funktionen von Pronomina und Vergleichspartikeln sowie die Rolle von Nebensätzen bei der Satzspaltung behandelt.

Relativa und Interrogativa müssen im Nebensatz aufgrund ihres Phrasenstatus das Vorfeld einnehmen. Die linke Klammerposition ist eine Kopfposition, in der sie nicht stehen können. $\mathrm{Zu}$ freien Relativsätzen können d-Pronomina als Antezedentien ergänzt werden. Diese fungieren als pronominale Bezugsausdrücke. Vorangestellte Nebensätze aller Art können (wie auch vorangestellte Ausdrücke anderer Klassen) durch koindizierte Resumptiva wiederaufgenommen werden. Solche Strukturen werden traditionell als „Linksversetzungen“ analysiert. Ich habe die alternative These vertreten, dass Antezedens und Resumptivum zusammen das Vorfeld einnehmen. Ausgeklammerten Nebensätzen kann im Matrixsatz ein koindiziertes Korrelatpronomen (es oder ein Pronominaladverb) vorangestellt werden, das die syntaktische Position anzeigt, in der der Nebensatz interpretiert werden soll.

Eine nur selten beachtete Klasse von Nebensatzeinleitern sind Vergleichspartikeln. Sie können Vergleichspartikelsätze, Komplementierersätze, Subjunktorsätze und V1-Nebensätze einleiten. Ferner können sie auch freien w-Relativsätzen und indirekten w-Fragesätzen 
Blühdorn, H. - Syntaktische Nebensatzklassen im Deutschen

vorangestellt werden. Das wurde in diesem Aufsatz aber nicht behandelt und deshalb in Abb. 15 auch nicht verzeichnet. Mit Vergleichspartikeln eingeleitete Nebensätze kommen in allen Satzgliedfunktionen vor. Eine wichtige Rolle spielen sie als Argumente zu Grad- und Vergleichsprädikaten. Die einzelnen Klassen von Prädikaten fordern unterschiedliche Vergleichspartikeln als Ergänzung.

Bei der Satzspaltung bildet die abgespaltene Konstituente typischerweise mit dem Kopulaverb sein den Matrixsatz; der Satzrest erhält die Form eines Nebensatzes. Bei der Abspaltung von Verbalphrasen wird der Satzrest ein freier w-Relativsatz in Subjektfunktion; bei der Rechtsabspaltung von Argumenten (im sogenannten Pseudospaltsatz) wird er ein freier w-Relativsatz in Prädikativfunktion; bei der Linksabspaltung von Argumenten wird er ein freier d-Relativsatz in Prädikativfunktion, bei der Abspaltung von Adverbialia ein dassSatz in Subjektfunktion.

\section{Literaturverzeichnis}

Altmann, Hans. Formen der „Herausstellung“ im Deutschen. Rechtsversetzung, Linksversetzung, Freies Thema und verwandte Konstruktionen. Tübingen: Niemeyer, 1981.

AUER, Peter. „Zwischen Parataxe und Hypotaxe: 'abhängige Hauptsätze' im Gesprochenen und Geschriebenen Deutsch“. In: Zeitschrift für germanistische Linguistik 26, 1998. S. 284-307.

BLÜHDORN, Hardarik. „Zur Struktur und Interpretation von Relativsätzen“. In: Deutsche Sprache 35, 2007. S. 287-314.

BLÜHDORN, Hardarik. „A semantic typology of sentence connectives“. In: Harden, Theo / Hentschel, Elke (Hg.). 40 Jahre Partikelforschung. Tübingen: Stauffenburg, 2010. S. 215-231.

BLÜHDORN, Hardarik. Negation im Deutschen. Syntax, Informationsstruktur, Semantik. Tübingen: Narr, 2012(a).

BLÜHDORN, Hardarik. „Verknüpfungseigenschaften von Satzkonnektoren im Deutschen. Am Beispiel der Kausal- und Konsekutivkonnektoren“. In: Deutsche Sprache 40, 2012(b). S. 193-220.

BLÜHDORN, Hardarik. „Zur Syntax adverbialer Satzverknüpfungen: Deutsch - Italienisch Portugiesisch“. In: Gunkel, Lutz / Zifonun, Gisela (Hg.). Deutsch im Sprachvergleich. Grammatische Kontraste und Konvergenzen. Berlin: de Gruyter, 2012(c). S. 301-331.

BLÜHDORN, Hardarik / LOHNSTEIN, Horst. „Verumfokus im Deutschen: Versuch einer Synthese“. In: Lohnstein, Horst / Blühdorn, Hardarik (Hg.). Wahrheit - Fokus - Negation. Hamburg: Buske, 2012. S. 171-261.

Diewald, Gabriele. Die Modalverben im Deutschen. Grammatikalisierung und Polyfunktionalität. Tübingen: Niemeyer, 1999.

DuDEN. Die Grammatik. Unentbehrlich für richtiges Deutsch. 8. Auflage. Mannheim: Dudenverlag, 2009. 
Blühdorn, H. - Syntaktische Nebensatzklassen im Deutschen

EISENBERG, Peter. Grundriß der deutschen Grammatik. Band 2: Der Satz. 2. Auflage. Stuttgart: Metzler, 2004.

ENGEL, Ulrich. Deutsche Grammatik. 3. Auflage. Heidelberg: Groos, 1996.

FABRICIUS-HANSEN, Cathrine. „Sogenannte ergänzende wenn-Sätze. Ein Beispiel semantischsyntaktischer Argumentation“. In: DYHR, Mogens / HYLDGAARD-JENSEN, Karl / OLSEN, Jørgen. (Hgg.). Festschrift für Gunnar Bech zum 60. Geburtstag. Kopenhagen: Institut for germansk filologi, 1980. S. 160-188.

FREY, Werner. „Peripheral adverbial clauses, their licensing and the prefield in German“. In: Breindl, Eva / Ferraresi, Gisella / Volodina, Anna (Hg.). Satzverknüpfungen. Zur Interaktion von Form, Bedeutung und Diskursfunktion. Berlin: de Gruyter, 2011. S. 41-77.

GÄRTNER, Hans-Martin. „Are there V2 relative clauses in German? "In: The Journal of Comparative Germanic Linguistics 3, 2001. S. 97-141.

HAEGEMAN, Liliane. „The syntax of adverbial clauses and its consequences for topicalization“.(2002). Online unter: http://webh01.ua.ac.be/apil/apil107/file05.pdf. (Letzter Zugang: 18/06/2013)

HAEGEMAN, Liliane. „Conditional Clauses: External and Internal Syntax“. In: Mind \& Language 18, 2003. S. 317-339.

HAEGEMAN, Liliane. „Conditionals, factives and the left periphery“. In: Lingua 116, 2006. S. 16511669.

HOLLER, Anke. Weiterführende Relativsätze. Empirische und theoretische Aspekte. Berlin: Akademie Verlag, 2005.

HOLLER, Anke. „Uniform oder different? Zum syntaktischen Status nicht-restriktiver Relativsätze“. In: Deutsche Sprache 35, 2007. S. 250-270.

Lehmann, Christian. Der Relativsatz. Typologie seiner Strukturen, Theorie seiner Funktionen, Kompendium seiner Grammatik. Tübingen: Narr, 1984.

PASCH, Renate / BRAUßE, Ursula / BREINDL, Eva / WABNER, Ulrich Hermann. Handbuch der deutschen Konnektoren: Linguistische Grundlagen der Beschreibung und syntaktische Merkmale der deutschen Satzverknüpfer (Konjunktionen, Satzadverbien und Partikeln). Berlin: de Gruyter, 2003.

REICHMANN, Tinka. Satzspaltung und Informationsstruktur im Portugiesischen und im Deutschen. Ein Beitrag zur Kontrastiven Linguistik und Übersetzungswissenschaft. Frankfurt/Main: Lang, 2005.

REIS, Marga / WÖLLSTEIN, Angelika. „Zur Grammatik (vor allem) konditionaler V1-Gefüge im Deutschen“. In: Zeitschrift für Sprachwissenschaft 29, 2010. S. 111-179.

SCHWARZ-FrIESEL, Monika / CONSTEN, Manfred / MARX, Konstanz. „Semantische und konzeptuelle Prozesse bei der Verarbeitung von Komplex-Anaphern“. In: Pohl, Inge / Konerding, KlausPeter (Hg.). Stabilität und Flexibilität in der Semantik. Strukturelle, kognitive, pragmatische und historische Perspektiven. Frankfurt/Main: Lang, 2004. S. 67-86.

STERNEFELD, Wolfgang. Syntax. Eine morphologisch motivierte generative Beschreibung des Deutschen. 2 Bände. 3. Aufl. Tübingen: Stauffenburg, 2008/2009.

Wöllstein-Leisten, Angelika / HeIlmann, Axel, / StePAn, Peter / Vikner, Sten. Deutsche Satzstruktur. Grundlagen der syntaktischen Analyse. Tübingen: Stauffenburg, 1997.

ZIFONUn, Gisela / HoFFMANN, Ludger / STRECKER, Bruno et al. Grammatik der deutschen Sprache. 3 Bände. Berlin: de Gruyter, 1997. 